\title{
Expérience radar Rodin sur les départements du Val de Marne et de Seine Saint-Denis
}

\section{The Rodin radar experiments in the Departments of Val de Marme and Seine Saint-Denis}

\author{
H. Andrieu* \\ LCPC, Paris
}

\author{
G. Jacquet et M. Torrelli \\ CERGRENE, Paris
}

\section{Introduction}

La notion de gestion automatisée d'un réseau d'assainissement répond à plusieurs soucis dont celui d'une meilleure utilisation des équipements existants pour limiter les risques d'inondation et de déversements polluants. Cependant pour être réalisable sur le plan opérationnel, la gestion automatisée doit s'appuyer sur l'acquisition, en temps réel, de données variées, et en premier lieu des mesures pluviographiques. De leur qualité dépend, en effet, la fiabilité de la prévision à court terme des débits à l'exutoire des bassins versants alimentant les collecteurs principaux.

Les moyens actuels de mesures pluviographiques, qui ont une bonne représentativité ponctuelle, ne permettent pas toujours de saisir les variations spatiales de la pluie. De plus, certains episodes pluvieux préjudiciables, d'origine convective peuvent avoir un développement très localisé (quelques $\mathrm{km}^{2}$ ). Seule une densification extrême des réseaux de mesures au sol, lourde en fonctionnement pallierait cet inconvénient.

Parallèlement la Météorologie Nationale a développé des systèmes radar d'acquisition de données dont l'intérêt a été multiplié par les techniques de numérisation d'images. Ces outils offrent donc une information nouvelle sur la répartition spatiale de la pluie et entrainent une collecte immédiate et centralisée des données. Par ailleurs, de nombreux travaux [1], [2], [3] ont montré l'intérêt du radar dans l'évaluation des lames d'eau moyennes. Prenant en compte ces divers éléments, l'étude entreprise est consacrée à l'exploitation à des fins hydrologiques, en milieu urbain, des données collectées au moyen d'un radar développé par la Météorologie Nationale. Elle devra, à terme, tenter d'évaluer l'intérêt de ce dernier dans le domaine de la simulation des débits et déboucher, en outre, à partir des résultats obtenus sur la définition des conditions d'utilisation des images radar.

\footnotetext{
* participe à cette étude du CERGRENE.
}

2. Expérience entreprise et données collectées

Durant les mois de juillet et août 1982 , les Services de la Météorologie Nationale (CTM de Trappes) ont effectué des enregistrements de séquences pluvieuses au moyen de la chaine RODIN [4] localisée à $22 \mathrm{~km}$, distance minimale, d'une zone d'étude qui regroupe les départements de la Seine St-Denis et du Val de Marne.

Cette zone (Fig. 1) dispose en outre d'un réseau dense de pluviographes enregistreurs (21 appareils plus trois stations météorologiques) transducteurs ou RMN 302 de "Précis Mécanique", vitesse de déroulement $30 \mathrm{~mm} / \mathrm{h}$ ou $20 \mathrm{~mm} / \mathrm{h}$. En complément, des limnigraphes suivent l'évolution des débits à l'exutoire de plusieurs bassins versants tests. Ces appareils sont installés et entretenus par des équipes de mesures spécialisées des Directions Départementales de l'Equipement. Ce suivi permet une grande fiabilité.

Il a ainsi été possible de recueillir des données sur cinq épisodes pluvieux d'origines météorologiques diverses.

- 13-14 juillet : très importantes cellules convectives se déplaçant,

- 21-22 juillet: enregistrements perturbés par des pannes de secteur,

- 30 juillet : situation frontale le matin, suivi en fin d'après-midi d'un essaim de cellules convectives locales.

- 2 août : cellules convectives locales.

Aucune de ces séquences pluvieuses n'a atteint une fréquence de retour élevée, le maximum enregistré localement se situant autour de $40 \mathrm{~mm}(21 / 07)$.

La principale difficulté a consisté à synchroniser les données radar et pluviographiques. Elle a été résolue par l'utilisation commune de l'horloge parlante comme référence de temps, et par une visite systématique sur les sites pluviographiques, immédiatement avant ou après 


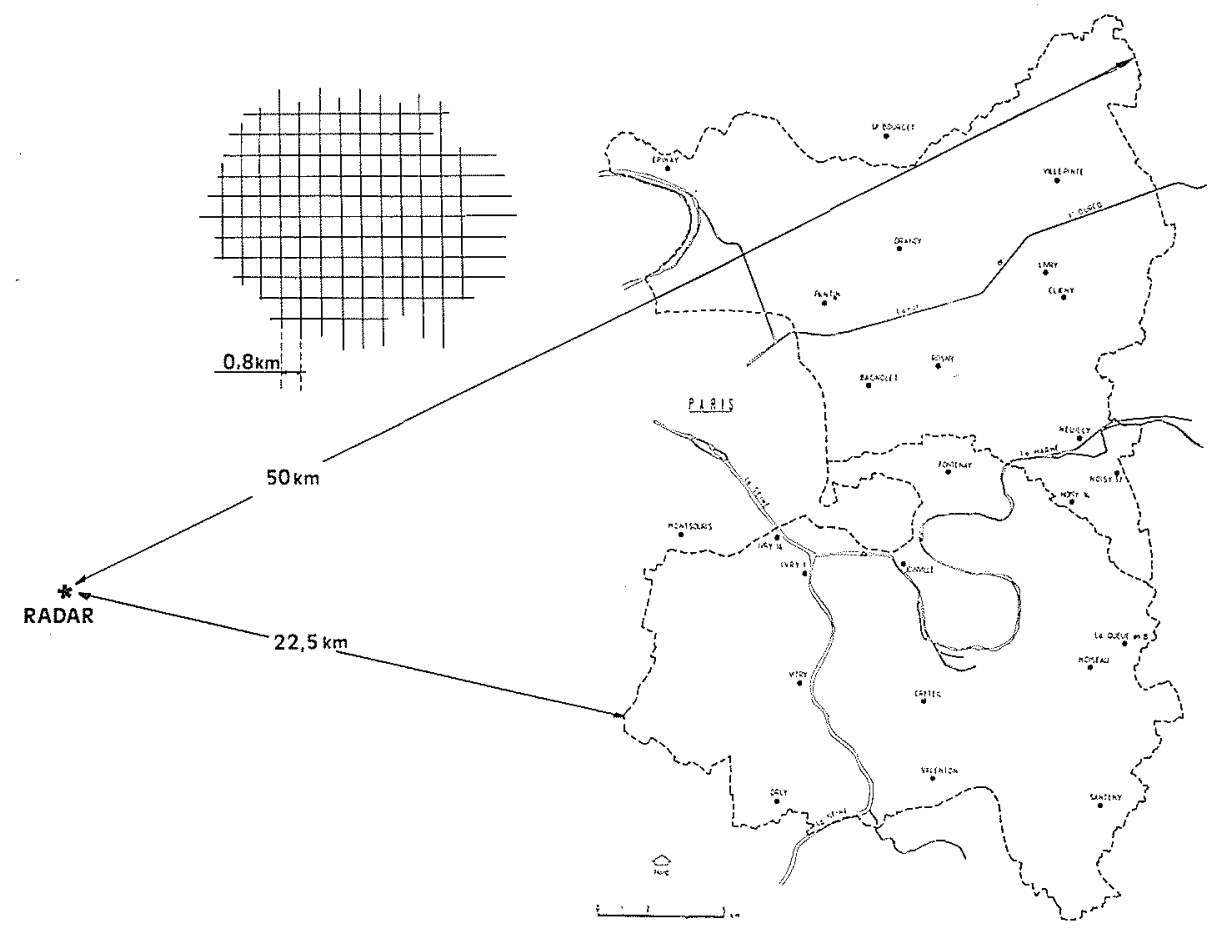

Figure 1 - Représentation géographique de la zone étudiée.

chaque séquence pluvieuse, pour effectuer un recalage horaire. $70 \%$ environ des enregistrements pluviographiques sont considérés comme fiables : erreur limitée à 2 ou 3 minutes compte tenu des précautions prises.

Parallèlement se pose, le problème de la représentativité des mesures de pluviographes à augets basculeurs, souvent mise en doute pour les fortes intensités. Une analyse sommaire de la répartition des intensités sur 5 minutes révèle que seulement $1,3 \%$ d'entre-elles sont supérieures à $50 \mathrm{~mm} / \mathrm{h}$. Les incertitudes de mesure notamment liées à la mise en charge de l'orifice du cône et aux pertes à chaque basculement d'augets [5] sont donc réduites.

Pour l'ensemble de ces séquences pluvieuses, nous disposons en outre d'images radar qui présentent les caractéristiques suivantes:

- angle de site du radar : $0,5^{\circ}$

- fréquence d'acquisition moyenne : une image toutes les 110 secondes.

- images maillées en coordonnées cartésiennes : taille de la maille, $800 \mathrm{~m}$ de côté.

- numérisation des valeurs de réflectivité en 16 niveaux:

4 dB d'amplitude environ par niveau.

Les principales sources d'incertitudes d'acquisition des données radar résident dans la discrétisation en niveaux. La grande fréquence d'acquisition des images nous offre cependant une remarquable couverture radar des séquences pluvieuses retenues.

\section{Images radar et hydrologie urbaine}

Les bassins versants capables de saturer des collecteurs principaux peuvent être de très petite superficie (généralement entre 100 ha et 200 ha en Seine St-Denis), ils sont donc couverts par deux ou trois pixels seulement. De plus, leur forte imperméabilisation, qui peut dépasser $30 \%$, contribue à diminuer un temps de réponse parfois inférieur à la demi-heure. La mise en œuvre de modèles de simulation du ruissellement nécessite donc un pas de temps de travail de 10 à 15 minutes.

La plupart des expériences consacrées jusqu'à maintenant à l'utilisation hydrologique du radar météorologique se sont intéressées à des surfaces beaucoup plus vastes et à des durées d'intégration dans le temps supérieures (l'heure ou la durée de l'averse).

Compte tenu de nos objectifs, l'utilisation que nous envisageons des images radar se situe dans des perspectives différentes :

- mise en ouvre de données quasi-ponctuelles.

- pas de temps court.

Nous sommes donc a priorité très exposés à tous les effets de l'instabilité de la rétrodiffusion radar.

Toutefois, les conditions expérimentales précédemment évoquées nous offrent quelques atouts non négligeables :

- la proximité du radar de la zone d'étude et l'absence de relief limitent l'importance de sources d'erreurs potentielles : atténuation du faisceau due à la distance ou derrière une cellule intense, perte de représentativité due à l'altitude ( $350 \mathrm{~m}$ environ dans notre cas).

En outre, l'évaluation des intensités pluvieuses ne constitue pour nous qu'un moyen. Nous devons notamment garder à l'esprit qu'autant qu'une restitution fidèle du déroulement de l'épisode pluvieux, nous souhaitons disposer d'une bonne estimation des débits, et en particulier des débits de pointe qui correspondent naturellement aux intensités les plus fortes. 

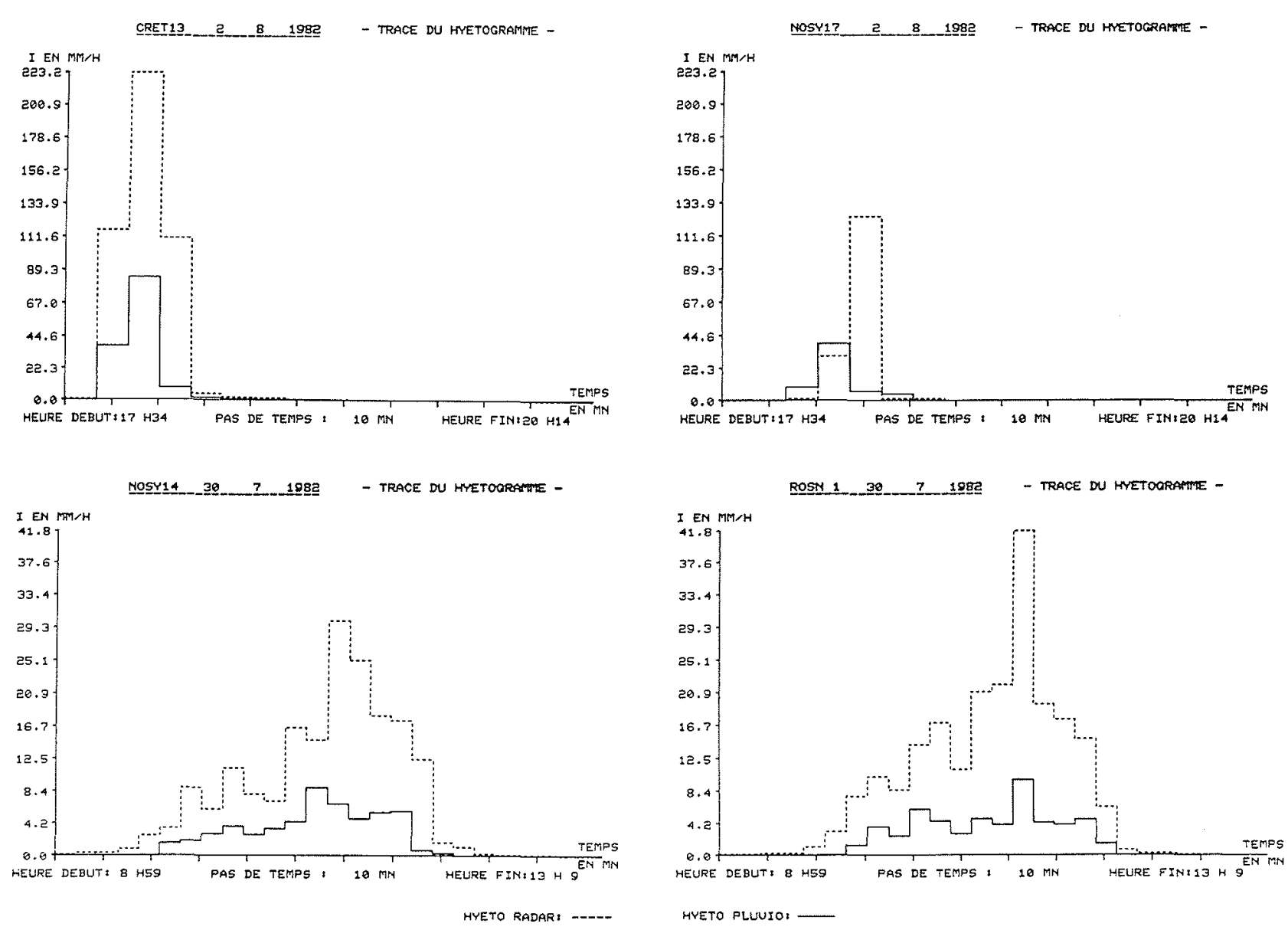

Figure 2 - Hyétogrammes issus des données radar brutes:

a) le 30 juillet 1982

b) le 2 août 1982

Enfin, compte tenu des données recueillies, une base de temps de travail de 10 ou $15 \mathrm{~min}$ semble raisonnable; descendre jusqu'à 5 min parait a priori plus risqué. Un compromis devra être trouvé entre la précision des résultats et la discrétisation dans le temps.

\section{Exploitations des données. Premières consta- tations.}

Ayant entrepris très récemment cette tâche, nous ne disposons encore que de très peu de résultats. Nous nous limiterons ici à une évocation qualitative de deux séquences pluvieuses.

- le 30 juillet au matin, d'origine frontale,

- le 2 août, convective.

Cette approche constitue toutefois une introduction aux divers problèmes posés par l'exploitation des images radar.

Nous supposerons également, pour représenter et comparer les résultats, que pluviographe et pixel radar qui le recouvre ont une représentativité équivalente sur un pas de temps de plusieurs minutes.

Nous avons retenu tous les pluviogrammes bien que quelques-uns soient peu sûrs.

Enfin, toutes les valeurs utilisées sont ponctuelles, ou distributions de valeurs ponctuelles (et non in tégrées sur les 1400 pixels de la zone d'étude).

\subsection{Données brutes}

Le cumul des images a été effectué (en attribuant à chaque niveau sa valeur médiane en termes de réflectivité) par l'intermédiaire d'une moyenne arithmétique sur les réflectivités. Compte tenu du système de marquage horaire du radar, les images situées dans une fourchette d'une minute de part et d'autre des limites de ce pas de temps, sont comptabilisées dans chacune d'entre eux.

Pour le 30 juillet, l'utilisation de la loi de MarshallPalmer $\left(Z=200 R^{1,6}\right)$, la plus courante pour une pluie frontale, a permis de passer aux intensités pluvieuses.

En ce qui concerne le 2 aout, la relation suivante [6], a été adoptée : $Z=486 R^{1,37}$

La figure 2 présente, pour ces deux dates, deux courbes comparatives en divers points pluviographiques.

\subsubsection{Le 30 juillet}

L'évolution des images restitue correctement l'avancée de la zone pluvieuse. Toutefois, on peut noter un décalage de 20 à $30 \mathrm{~min}$ environ entre l'apparition des premiers échos radar, faibles, et le début estimé de la 

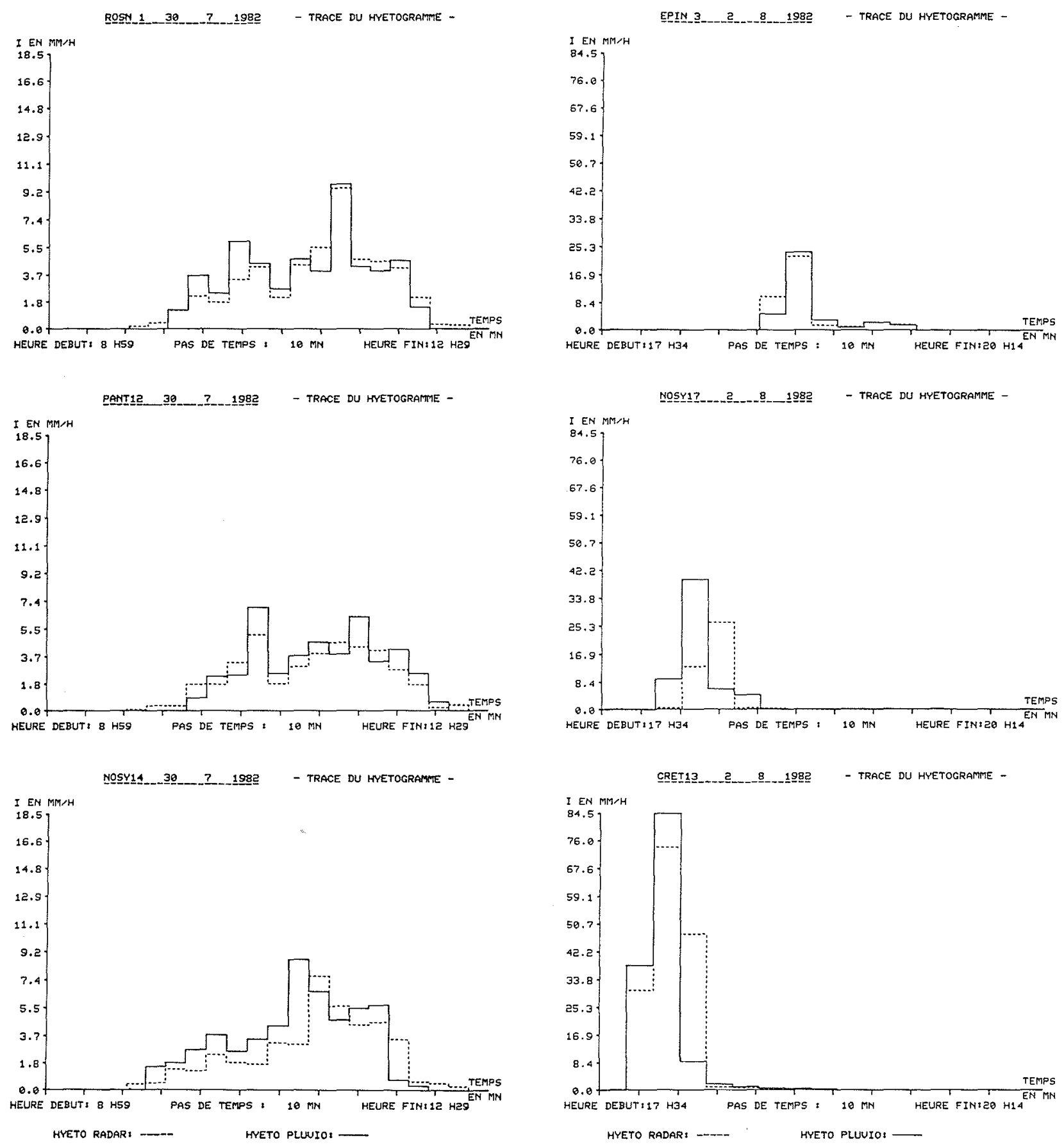

Figure 3 - Hyétogrammes issus des données radar corrigées. a) le 30 juillet 1982 .

pluie aux pluviographes. On peut émettre l'hypothèse que les très faibles in tensités du front mettent un certain temps à mouiller le cone et à remplir l'auget.

La principale constatation reste néanmoins la très forte surestimation des intensités, entrainée par la loi de Marshall-Palmer (le rapport moyen pluie-pluviographe sur pluie-radar vaut environ 0,28 ), toutefois la forme générale du hyétogramme reste convenablement restituée.

\subsubsection{Le 2 août}

Dans ce cas également, la surestimation des intensités

par le radar est sensible. Toutefois, à la différence de l'exemple précédent, la restitution des hyétogrammes ponctuels semble beaucoup plus irrégulière, notamment pour un groupe de stations (Neuilly - Noisy 14 et 17 et Rosny principalement) où le radar "voit" mal et sousestime fortement les intensités pluvieuses pendant une durée de 20 minutes (identique pour toutes). Ce phénomène ne semble pas pouvoir s'expliquer par la présence de cellules intenses sur le trajet du faisceau et susceptibles de provoquer une telle atténuation du signal. Peut être faut-il rechercher les causes de cette sous-estimation dans l'hétérogénéité de la pluie en tête de cellules? 
Ces premières observations confirment la méfiance, notée dans la littérature, à entretenir vis-à-vis d'une u tilisation des données radar brutes à travers une loi moyenne.

\subsection{Données corrigées}

Nous nous sommes, dans une première phase, limités à une méthode de correction qui conserve l'in tégrité des données radar, notre principal objectif restant à ce stade de "prendre connaissance" de ces dernières en les mettant en parallèle avec des données pluviographiques. Nous n'avons pas jugé nécessaire, pour cette approche, l'utilisation de pluviographes de vérification.

Il s'agit simplement d'introduire un facteur de correction moyen par pas de temps $\left(f_{k}\right)[6]$ de la forme :

avec :

$$
f_{k}=\frac{\sum_{i}^{n} P_{i k}}{\sum^{n} R_{i k}}
$$

$k \quad=$ indice du pas de temps

$P_{i k}=$ intensité pluviographique au point $i$

$R_{i k}=$ estimation de l'intensité radar brute en $i$

$n$ = nombre de points de comparaison.

d'où l'on déduit une intensité radar corrigé en $i\left(R C_{i k}\right)$ :

$$
R C_{i k}=f_{k} \times R_{i k}
$$

La figure 3 présente des exemples d'application de cette méthode. Elles confirment principalement que, si le 30 juillet, la reproduction des courbes d'intensité pluvieuse au moyen de données radar corrigées est correcte et parfois très bonne, le 2 août, les résultats sont beaucoup plus contrastés et parfois incohérents. Nous reviendrons ultérieurement sur cette nette différence entre les deux séquences pluvieuses.

\subsubsection{Description de la dispersion des écarts}

Pour tenter de quantifier ces impressions et d'estimer la dispersion des écarts, nous avons utilisé l'indicateur $E$ de la forme:

$$
E=\frac{\sum_{i, k}\left|P_{i k}-R C_{i k}\right|}{\sum_{i, k} P_{i k}}
$$

qui apprécie l'écart moyen, pondéré par l'intensité de la pluie (rappelons que nous accordons une importance plus grande aux fortes intensités); $E$ peut être évalué par station, par pas de temps ou sur la totalité des données disponibles.

\section{a) influence du pas de temps}

Les courbes Figure 4, représentent l'influence du pas de temps de cumul sur l'écart moyen estimé avec la totalité des données pour chacune des averses.

Des durées de $5,10,15,20$ et 30 min ont été prises

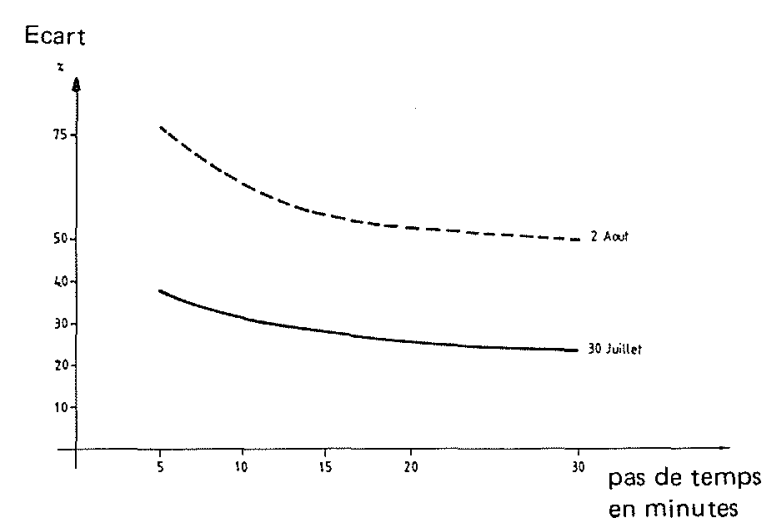

Figure 4 - Evolution de l'écart en fonction du pas de temps de cumul.

en compte. Ces courbes confirment d'abord que, dans tous les cas, nous obtenons une meilleure restitution des hyétogrammes, le 30 juillet que le 2 août.

Il apparait également que conformément aux résultats bibliographiques, l'allongement du pas de temps se traduit par une réduction des écarts entre les indications pluviographiques et radar.

b) pluie et ruissellement

Cependant un allongement de la discrétisation en temps se traduit par une perte de représentativité de la simulation des débits à l'exutoire des bassins versants urbains critiques (30 à $45 \mathrm{~min}$ de temps de concentration). De plus, un bassin versant constitue, relativement à l'intensité de la pluie, un filtre basse fréquence, susceptible de lisser en partie les incertitudes des données, dans la mesure toutefois où la distribution des écarts demeure aléatoire au cours du temps, point qui reste à confirmer.

A titre d'exemple, nous avons positionné un bassin fictif de 200 ha, imperméabilisé à $20 \%$ et admis un temps de concentration de $40 \mathrm{~min}$ sous chaque pluviographe en supposant par ailleurs qu'un modèle linéaire simple représentait parfaitement sa réponse. Ce bassin a été soumis aux impulsions pluviographique et radar corrigé.

Pour un pas de temps de $10 \mathrm{~min}$, il apparait que l'écart moyen sur les estimations de pluie qui est de 0,32 pour le 30 juillet et de 0,61 pour le 2 août devient respectivement de 0,19 et 0,37 pour les débits.

Cet exemple fictif, illustre l'influence possible d'un modèle de ruissellement sur la réduction des écarts.

Enfin, il est à noter que si l'on prend en compte la lame d'eau totale tombée en chaque point, la différence entre les estimations n'est plus que de $11 \%$ pour le 30 juillet et de $35 \%$ pour le 2 août, elle reste indépendante du pas de temps.

Si les résultats de ce calibrage ne paraissent pas pleinement satisfaisants pour le 2 août, ils sont très encourageants pour le 30 juillet.

\subsection{0 juillet et 2 août. Pourquoi de telles différences?}

Nous ne disposons actuellement pas d'éléments décisifs permettant de justifier les différences dans la qualité de restitution des deux averses. 

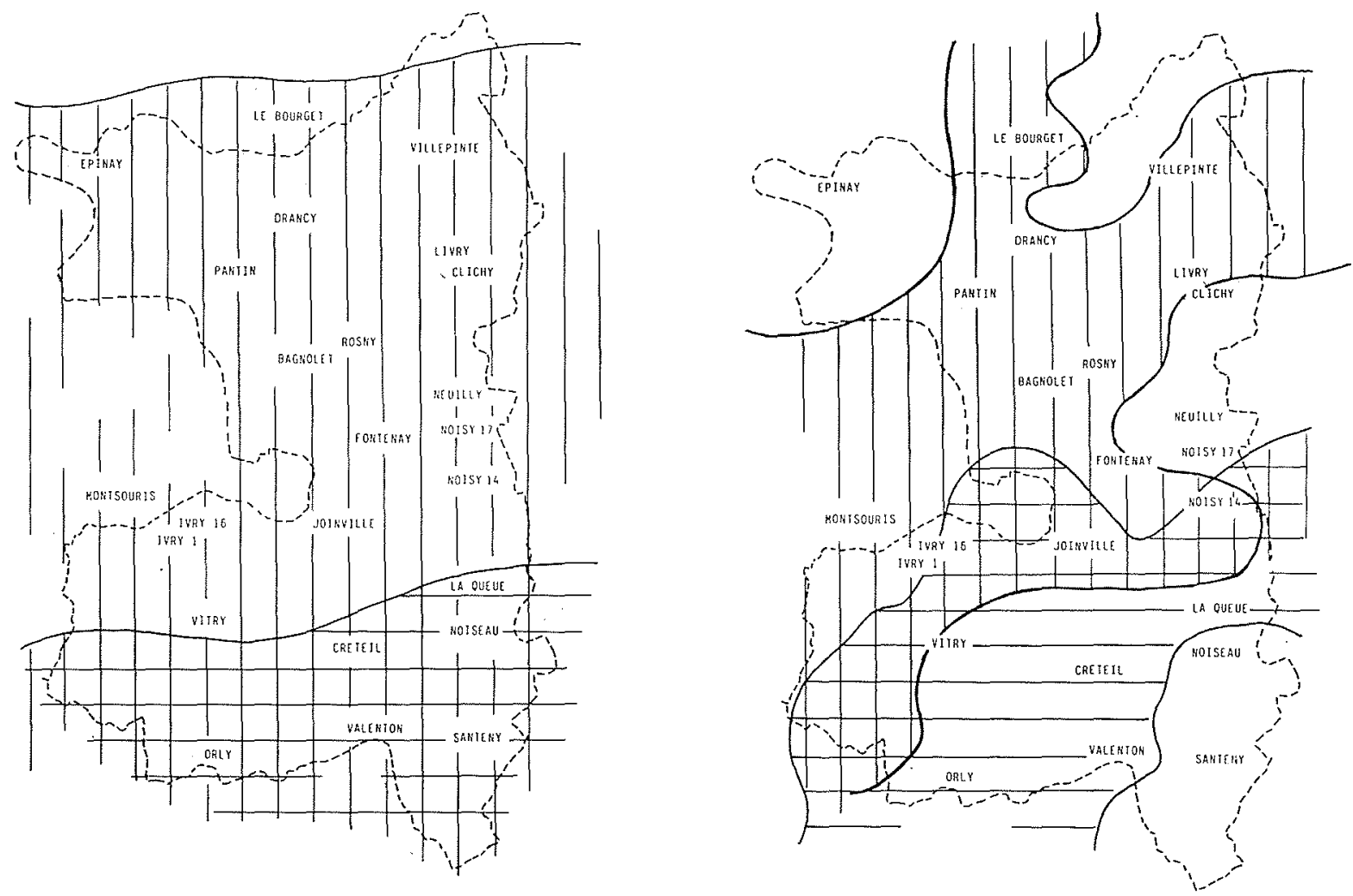

Secteurs atteints par la pluie

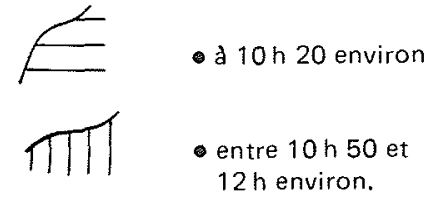

$3,17 \mathrm{~mm} / \mathrm{h}$ $0,60 \mathrm{~mm} / \mathrm{h}$

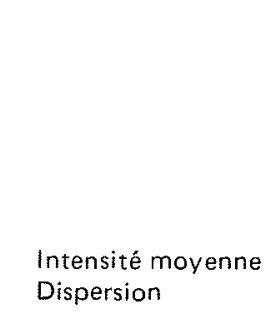

: $6,30 \mathrm{~mm} / \mathrm{h}$

: $3,82 \mathrm{~mm} / \mathrm{h}$

Figure 5 - Déroulement des séquences pluvieuses d'après les images radar.

a) le 30 juillet 1982

b) le 2 août 1982 .

Toutefois, une explication peut être avancée en considérant leur diversité d'origine et de structure :

- le 30 juillet, au matin, la situation frontale s'est traduite par des pluies régulières et continues, réparties de façon homogène sur l'ensemble de la zone d'étude.

- lors du 2 août, au contraire, cette surface a été touchée de manière très diversifiée par des cellules convectives pratiquement distinctes et susceptibles de présenter des caractéristiques différentes.

La figure 5, qui restitue schématiquement le déroulement des deux averses, sur la zone d'étude, souligne cette diversité ; contrairement au 30 juillet, le 2 août il ne pleut jamais sur l'ensemble de cette surface. De plus, la dispersion des intensités moyennes, beaucoup plus large le 2 août que le 30 juillet, traduit l'hétérogénéité de cette pluie.

Par ailleurs, nous avons, pour cette première phase, utilisé une méthode de calibrage basée sur un facteur de correction moyen appliqué à toute la zone d'étude. Cette approche prend mal en compte les singularités locales des précipitations, beaucoup plus probables dans un cas de convection locale comme le 2 août que dans une situation frontale telle que le 30 juillet.

Les écarts enregistrés entre les deux séquences pluvieuses pourraient donc s'expliquer par l'inadaptation d'un tel facteur de correction aux pluies hétérogènes; ceci reste toutefois à préciser et à confirmer sur les épisodes restant à exploiter, dont certains présentent un caractère convectif affirmé.

\section{Conclusion}

Il est difficile, au vu de ces premiers éléments, de tirer des conclusions quant à l'utilisation du radar pour une meilleure connaissance spatiale de la plu ie à l'échelle des bassins versants urbains. Toutefois, les premiers 
résultats obtenus sur les séquences du 30 juillet, sont très encourageants, et permettent d'espérer que l'utilisation combinée du réseau pluviographique et du radar assurera une précision suffisante pour les besoins d'une gestion automatisée. Néanmoins, l'averse convective du 2 août tend à montrer que pour des pas de temps très courts, l'hétérogénéité spatiale, sur quelques centaines de $\mathrm{km}^{2}$, doit être prise en considération. Nous nous orientons donc vers' un calibrage des images radar qui intègre cet élément, l'aspect complémentaire des indications radar et pluviographique prenant alors toute sa signification.

\title{
BIGLIOGRAPHIE
}

[1] Rapport Hydromel. Météorologie Nationale. 1979.

[2] Dee Weather Radar and Ral Time Hydrological Forecasting Project. Central Water planning Unit. Nov. 1977.

[3] Radar Rainfall Measurement. A Summary Wilson. Brandes. Bulletin American Meteorological Society Vol. 60, $\mathrm{n}^{\circ} 9$, Septembre 1979.

[4] Principe de fonctionnement des radars météorologiques. David. CTM.

[5] Le tarage des pluviographes. Oberlin. Galéa, Rosique. La météorologie (Mars et Juin 1980).

[6] Radar Météorologie Sauvageot. Eyrolles.

\section{Discussion}

\author{
Président : J. ESTIENNE
}

Rappelant le problème du recalage de l'information radar sur l'information pluviographique, $M$. LE QUENTREC demande si un recalage permanent dans le temps et dans l'espace améliorerait considérablement la représentation de l'averse, en s'affranchissant des problèmes de masque ou de variation de site. Il voit dans ce recalage permanent une perspective intéressante, dans la mesure où la concentration simultanée des informations radar et pluviographiques télétransmises permet une reconstitution très fidèle des pluviogrammes.

M. ANDRIEU reconnaît que les conditions de l'expérience ont été favorables et qu'il n'y avait pas eu de masque. Les expériences vont se poursuivre tout en conservant un objectif réaliste pour ne pas avoir des temps de calcul trop longs. On ne sait pas encore si le recalage permanent sera effectué par "pas de temps" ou par une autre méthode, mais il faudra bien passer par un recalage permanent.

M. ROBERT s'enquérant de la possibilité d'utiliser le procédé pour la gestion opérationnelle d'un réseau d'assainissement, $M$. ANDRIEU précise que le passage aux débits n'a pas encore été étudié, mais il pense que l'on devrait aboutir, par un modèle de ruissellement, à opérer un certain filtrage qui permettrait de mieux reproduire les débits.

M. $J A C Q U E T$ fait observer que les résultats obtenus ont été remarquables et que, par conséquent, les valeurs qui seront introduites dans un modèle de simulation présenteront une incertitude nettement inférieure à ce qui est nécessaire.

M. BRIAT constate que ce modèle revient à caler le paramètre de la formule de Marshall-Palmer ; mais serait-il possible de caler également le second paramètre, variable d'une pluie à l'autre, pour améliorer la précision?

M. JACQUET répond que le second paramètre a une influence moindre que le premier, mais il est possible de modifier le paramètre exposant de la formule monome en fonction du type de pluie (convection frontale).

M. ROCHE estime que les fluctuations du rapport entre la pluie estimée et la pluie observée se présentent de telle façon qu'il semble difficile d'avoir en temps réel une bonne approxi- mation. D'un pas de temps sur l'autre, les fluctuations liées aux problèmes de mesures de radar ne sont pas graves, mais il y a néanmoins des risques de temps de réponse de filtrage qui peuvent conduire à des sous-estimations des intensités de toute une période de début d'averse.

M. JACQUET note que l'on parle actuellement de recalage spatial mais qu'il y a deux phénomènes. D'abord un recalage en temps réel : dans celui-ci, à chaque instant, on peut se trouver en début ou en fin d'une pluie; si les pluviographes sont nombreux, il s'en trouvera toujours un assez proche du début de la pluie. Mais il y a aussi le recalibrage spatial : cela consiste à utiliser le pluviographe le plus proche de la cellule, pour définir la correction qu'il faut donner à la valeur radar. Une autre possibilité consiste à suivre une cellule avec les pluviographes et de recalibrer en fonction de la cellule sur laquelle on se trouve.

M. FLOCH ayant évoqué le problème de l'organisation entre les différents services, $M . J_{A C Q U E T}$ estime qu'elle se fera avec le temps : on progresse par étapes. Lorsqu'on aura une prévision de la pluie plusieurs heures à l'avance, on pourra gérer beaucoup mieux le réseau, mais cette gestion peut déjà être améliorée dès la connaissance d'une détection de pluie. Les autres étapes suivront.

En tant qu'utilisateur de ces études, notamment en SeineSaint-Denis, $M$. BACHOC adresse ses remerciements aux participants de ces études, et plus particulièrement à M. DAVID qui a réalisé une performance pour les rendre possibles. Il demande ensuite aux responsables de la Météorologie si le radar de Trappes, qui a montré toutes ses qualités, pourrait voir son utilisation transformée par une application orientée vers les services d'assainissement de la région parisienne.

M. GILET répond que cela pourra être envisagé dès que le radar de Trappes deviendra opérationnel, mais cela entrânera des conséquences financières pour la maintenance et le développement du radar, estimées à un montant de l'ordre du million de francs. Il n'est donc pas possible de fixer une date, mais des essais de transmission à partir du radar pourraient être entrepris d'ici un an ou deux. 


\section{Abstract \\ The Rodin radar experiment in the Departments of Val de Marne and Seine Saint-Denis}

Automated operation of a sewerage system is based in particular on acquisition in real time of rain-gauge recordings required to evaluate direct water increments. In this context a digital picture meteorological radar gives a three-dimensional representation of the rain as a complement to the rain-gauge recordings. The current experiment intends to evaluate the merits involved. It is based on a measurement campaign carried out in Seine Saint-Denis and Val de Marne in conjunction with the National Meteorological Office.

Synchronous radar data, rain-gauge (20 stations over $450 \mathrm{~km}^{2}$ ) and water-level recordings were collected for six rainfalls of different origins. They were utilized with allowances made for the objectives to be obtained which impose constraints on application of the radar data: random use at short intervals ( 10 to $20 \mathrm{~min}$.).

Initial, incomplete results relate to a frontal rain and convective cells. They show that primary laws cannot be adapted: $\mathrm{Z}=A \mathrm{R}^{\mathrm{b}}$. Application of a simple correction method has given encouraging results for frontal rain but has proved unsuitable for convective rain which requires use of a calibration where local factors are taken into account. 